\title{
Hypoxia-Related Long Non-Coding RNA Signature Predicts the Prognosis of Breast Cancer
}

\section{Jinlong Huo}

The Third Affiliated Hospital of Zunyi Medical University (The First People's Hospital of Zunyi)

\section{Shuang Shen}

The Third Affiliated Hospital of Zunyi Medical University (The First People's Hospital of Zunyi)

\section{Chen Chen}

The Third Affiliated Hospital of Zunyi Medical University (The First People's Hospital of Zunyi)

\section{Rui Qu}

The Third Affiliated Hospital of Zunyi Medical University (The First People's Hospital of Zunyi)

\section{Youming Guo}

The Third Affiliated Hospital of Zunyi Medical University (The First People's Hospital of Zunyi)

Dong Ou (D1974456008@qq.com)

The Third Affiliated Hospital of Zunyi Medical University (The First People's Hospital of Zunyi)

\section{Research Article}

Keywords: Breast cancer, Hypoxia, TCGA, IncRNAs

Posted Date: November 22nd, 2021

DOI: https://doi.org/10.21203/rs.3.rs-1084000/v1

License: (c) (i) This work is licensed under a Creative Commons Attribution 4.0 International License.

Read Full License 


\section{Abstract}

Background: Breast cancer(BC) is the most common tumour in women. Hypoxia stimulates metastasis in cancer and is linked to poor patient prognosis.

Methods: We screened prognostic-related IncRNAs(Long Non-Coding RNAs) from the Cancer Genome Atlas (TCGA) data and constructed a prognostic signature based on hypoxia-related IncRNAs in BC.

Results: We identified 21 differentially expressed IncRNAs associated with BC prognosis. Kaplan Meier survival analysis indicated a significantly worse prognosis for the high-risk group $(P<0.001)$. Moreover, the ROC-curve (AUC) of the IncRNAs signature was 0.700 , a performance superior to other traditional clinicopathological characteristics. Gene set enrichment analysis (GSEA) showed many immune and cancer-related pathways and in the low-risk group patients. Moreover, TCGA revealed that functions including activated protein C (APC)co-inhibition, Cinnamoyl CoA reductase(CCR),check-point pathways, cytolytic activity, human leukocyte antigen (HLA), inflammation-promotion, major histocompatibility complex (MHC) class1, para-inflammation, $T$ cell co-inhibition, $T$ cell co-stimulation, and Type $\mathbb{B}$ and $\mathbb{Z}$ Interferons (IFN) responses were significantly different in the low-risk and high-risk groups. Immune checkpoint molecules such as ICOS, ID01, TIGIT, CD200R1, CD28, PDCD1(PD-1), were also expressed differently between the two risk groups. The expression of m6A-related mRNA indicated that YTHDC1, RBM15, METTL3, and FTO were significantly between the high and low-risk groups.Additionally, immunotherapy in patients with $B C$ from the low-risk group yielded a higher frequency of clinical responses to anti-PD-1/PD-L1 therapy or a combination of anti-PD-1/PD-L1 and anti-CTLA4 therapies.Except for lapatinib, the results also show that a high-risk score is related to a higher halfmaximal inhibitory concentration (IC50) of chemotherapy drugs.

Conclusion: A novel hypoxia-related IncRNAs signature may serve as a prognostic model for BC.

\section{Introduction}

$\mathrm{BC}$ is a highly lethal tumour in women, recently with an increasing incidence ${ }^{1,2}$. Approximately 1.7 million women are diagnosed with BC worldwide per year,and half a million women die from it each year ${ }^{3,4}$. The occurrence and development of $\mathrm{BC}$ is a very complicated pathological process, and the accurate pathogenesis of $\mathrm{BC}$ is still not clear ${ }^{5}$.In recent years, treatments for $\mathrm{BC}$ patients, such as surgical resection, radiation therapy, chemotherapy, hormone therapy, and targeted therapy have greatly improved patient progression-free(PFS)and overall survival(OS) ${ }^{6-8}$. However, metastasis and recurrence are still critical clinical events in $\mathrm{BC}^{9}$. Early detection of cancer by successful diagnostic markers improves the survival rate of patients with $\mathrm{BC}^{10}$. Currently, there is a lack of effective biomarkers for the early diagnosis of cancers ${ }^{11}$. However, in recent years, studies on the involvement of hypoxia in tumours have gradually begun to show that it is a hallmark of the tumour microenvironment. Tumors frequently develop under hypoxic conditions because of insufficient blood supply ${ }^{12}$. Severe tumour hypoxia ultimately leads to tissue necrosis, but hypoxia also exerts strong selective pressure towards a more aggressive tumour 
type ${ }^{13,14}$. Moreover, it is also reflected in enhanced angiogenesis and vasculogenesis reflecting altered gene expression, with suppression of apoptosis, promotion of autophagy ${ }^{15,16}$, stimulation of the epithelial-mesenchymal transition (EMT) ${ }^{17,18}$, and changes in anabolic phenotype to core cellular metabolism ${ }^{19,20}$. In addition, hypoxia is associated with increased DNA damage and reduced expression of various DNA repair proteins ${ }^{21-23}$. Meanwhile, hypoxia also promotes tumour immune evasion via diverse mechanisms ${ }^{24}$. All of these findings demonstrate that hypoxia in tumours is associated with a poor prognosis.

LncRNAs are a new type of potential biomarker and therapeutic target for cancer treatment ${ }^{25,26}$. Apart from gene regulation, a recent study found that IncRNAs participate in tumour invasion and metastasis by regulating the $\mathrm{EMT}^{27}$. For instance, the high expression of IncRNA p10247 is closely related to metastasis in breast cancer ${ }^{28}$. There is increasing evidence that various of IncRNAs can be utilized to construct a predictive model of the prognosis of different cancers ${ }^{29-31}$. Zhang et al. developed an effective hypoxia-related IncRNA assessment model for prognosis of clear cell renal carcinoma ${ }^{32}$. A study assembled 10 hypoxia-related IncRNAs by Zhang et al revealed that NOX4, CHST1 and COL8A1 were related to poor prognosis in human gastric cancer tissues ${ }^{33}$.In a related study, Gong et al. chose 13 hypoxia-related gene expression signatures to evaluate the hypoxic status of $B C$, indicating that BCL11A and CPEB2 might act as a oncogene and tumour suppressor, respectively ${ }^{34}$. But at the moment, there has been little research on hypoxia-related IncRNAs in BC. In the present study, we first identified 21 hypoxia-related IncRNAs and constructed a reliable prognostic model for further research in BC based on TCGA datasets using bioinformatics analysis. In addition, We then explored the role of hypoxia-related mRNA, immune responsesand chemotherapy efficacy in patients with invasive breast carcinoma.

\section{Results}

\section{Enrichment analysis of hypoxia-related genes}

We identified 69 hypoxia-related DEGs (43 downregulated and 26 upregulated; Table S2). Biological Process (BP), Molecular Function (MF), and Cellular Component (CC) were then considered. Kyoto Encyclopedia of Genes and Genomes (KEGG) -based analysis revealed that the over-expressed genes were mainly involved in the hypoxia-inducible factor (HIF)-1 signaling pathway, Glycolysis/Gluconeogenesis, MAPK signaling pathway, Insulin resistance and others (Figure 2 and Table S3).

\section{The hypoxia-related IncRNA prognostic signature}

We identified 3695 hypoxia-related IncRNAs (Table S4). Univariate COX analysis confirmed 274 significant hypoxia-related IncRNAs, which were included in the multivariate analysis.Overall, 21 differently expressed IncRNAs (AC087239.1,AC015802.3,GORAB-AS1,LINC01235,USP30AS1,LINC01614,LINC02446,AC121247.1,TFAP2A-AS1,LINC01929,KLHDC7B- 
DT,LINC00702,AL133467.1,TDRKH-AS1,AC004585.1,SIDT1-AS1,AC012213.3,HSD11B1-AS1,MIR44352HG,AL645608.7 and LRRC8C-DT)were found to be independent prognosis predictors in BC patients (Table S5).

\section{Survival results and multivariateanalysis}

Kaplan Meier survival analysis indicated a worse prognosis for the high-risk group $(P<0.001$, Figure $3 \mathrm{~A}$ ).Meanwhile, for predicting the prognosis of BC patients(Figure 2B) \the AUC of the IncRNA signature was 0.700 , showing superior performance to other traditional clinicopathological characteristics (Figure 3B).

As shown in our risk survival status plot, we found the risk score in $\mathrm{BC}$ patients increased proportionally to the survival of patients. Meanwhile,the heatmap showed 5 positive correlations and 8 negative correlations among the novel IncRNAs identified in this study, which needs validation in further work (Figure 3D).

The AUCs for 3-, 5-, and 7-year survival of predictions for the 21 IncRNA signature were $0.695,0.700$ and 0.713, respectively (Figure3C). According to COX analysis, age (HR:1.05, 95Cl: 1.03-1.07)and our risk signature (HR: 1.17, 95Cl: 1.10-1.23) were independent prognostic factors for survival of BC patients, respectively (Figure 4A, 4B). We can see the relationship between novel IncRNAs and mRNA from Figure 4C. In addition,The heatmap showing clinicopathological manifestations and hypoxia-related IncRNA prognostic signatures is also shown (Figure 5). Based on the relationship between clinicopathological characteristics and the selected hypoxia-related IncRNA prognostic signature, the clinical utility of the nomogram was accurate and steady and could be adopted for clinic management for BC patients (Figure 6).

\section{Gene set enrichment analyses}

The majority of the novel hypoxia-related IncRNAs in the prognostic signature in the low risk group was based on GSEA identified immune pathways such as asthma, autoimmune thyroid disease, T/B cell receptor signaling, FC gamma R-mediated phagocytosis, graft versus host disease, hematopoietic cell lineage, systemic lupus erythematosus, the intestinal immune network for IgAproduction, and primary immunodeficiency(Figure 7and Table S6).

\section{Immunity and gene expression}

Cell type Identification By Estimating Relative Subsets Of RNA Transcripts(CIBERSORT), ESTIMATE, Microenvironment Cell Populations-Counter method (MCP counter),single-sample gene set enrichment analysis (ssGSEA) and Tumor Immunoassay Resource (TIMER) algorithms were used to generate a heatmap of immune responses (Figure 8).Correlation analysis among immune cell subpopulations based on ssGSEA of TCGA-BRCA data indicated that T cell functions including APC co-inhibition, CCR,checkpoint expression, cytolytic activity, HLA, inflammation-promoting, MHC class1, para-

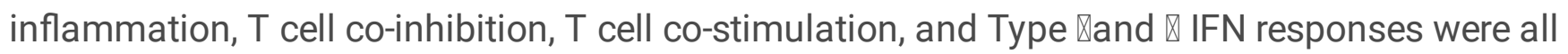


significantly different between the low-risk and high-risk groups (Figure 9A).Moreover,given the importance of checkpoint-based immunotherapy, we further explored the differences in immune checkpoints between the two groups.Significant differences in the expression of ICOS, ID01, TIGIT, CD200R1, CD28, PDCD1(PD-1) and other immune checkpoints between the two groups of patients are depicted in Figure 9B.The expression of m6A-related mRNA between the high and low-risk groups indicated that the expression of YTHDC1, RBM15, METTL3, and FTO wassignificant (Figure 10).Furthermore, to determine the capacity of the hypoxia-related IncRNA prognostic signature to predict the response to anti-PD-1/PD-L1 therapy, we used immunophenogramanalysis to investigate the correlation between the immunopheno-score (IPS) and low/high risk groups. We found that in the CTLA4_negative/positive + PD-1_negative/positive type, the low risk groupexhibited higher IPS than the high risk group ( $\mathrm{P} \otimes 0.0001)$ (Figure 9C). These results illustrate that patientsfrom the lowrisk group showed a higher positive response to anti-PD-1/PD-L1 therapy or a combination of anti-PD-1/PD-L1 and antiCTLA4 therapies.

\section{Correlation analysis between the risk model and chemotherapy outcome}

We also explored the relationship between the risk signature and the efficacy of common chemotherapeutic agents in the treatment of patients with BC based on the TCGA-BRCA project. We found that the high-risk score is related to a higher IC50 of chemotherapy drugs such as paclitaxel『p》 $0.01 \rrbracket$, camptothecin $(p<0.001)$, docetaxel $(p<0.05)$, epothiloneB $(p<0.05), A Z D 2281$ (Olaparib)) (

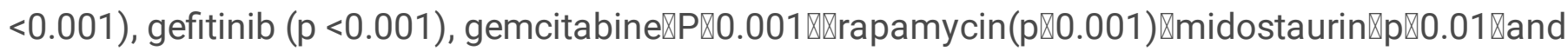
tipifarnib $(p<0.001)$, while the IC50 of lapatinib was found to be lower $(p<0.001)$. These findings indicate that the model can be used as a potential predictor of chemotherapeuticdrugsensitivity (Figure 11).

\section{Discussion}

Increasing evidence is indicating that tumour hypoxia may promote invasive cancer growth and metastasis ${ }^{45-47}$. Besides standard prognostic factors such as tumour stage, nodal status and tumour grade, tumour hypoxia in some cancers has been recommended as a prognostic factor for patient outcome ${ }^{48}$.In the present study, we identified novel hypoxia-related prognostic IncRNA signatures based on the TCGA-BRCA project. Subsequently, we have also explored the roles of immune infiltrating cells in the tumour microenvironment and the immune checkpoint inhibitors in patients with breast cancer. In addition, we provide evidence for potential biomarker and therapeutic targets in hypoxia signaling pathways.

In the present work, we identified 69 hypoxia-related DEGs. KEGG pathway analysis has further clarifiedthat these genes mainly participated in the hypoxia-inducible factor (HIF)-1 signaling pathway, Glycolysis/Gluconeogenesis, MAPK signaling pathway, insulin resistance and other signaling pathways. Guo et al. reported that HIFs targeta wide range of genes which function to control a variety of signaling 
pathways ${ }^{49}$;HIFs are expected to become new targets for hepatocellular carcinoma therapy .A recent study revealed that HIFs are the major sensors of low oxygen involved in viral replication and virion assembly ${ }^{50}$. In the present study, 21 differently expressed IncRNAs can be regarded as independent prognosis factors for BC patients. A recent study found that LINC01235 was upregulated in gastric cancer(GC) tissues and cells, and microRNA (miR)-6852-5p might be a key gene involved in the regulation of TWIST2 by LINC01235. The LINC01235-TWIST2 positive feedback loop mainly affected the migration and invasion of GC cells ${ }^{51}$. Similar studies showed that Immune-Related IncRNA USP30-AS1 was found to be over-expressed in cervical cancer, melanoma, glioblastoma multiforme and bladder cancer, associated with longer OS ${ }^{52-55}$. High LINC01614 expression was also markedly associated with the epithelial-mesenchymal transition (EMT), influencing metastasis and the prognosis of several cancers ${ }^{56}$.LncRNA LINC02446 suppresses the proliferation and metastasis of bladder cancer cells by binding with EIF3G and regulating the mTORsignaling pathway ${ }^{57}$. Results from a related study suggest that TFAP2A-AS1 acts as a tumour suppressor in BC via the miR-933/SMAD2 axis ${ }^{58}$.IncLINC01929 accelerates the progression of oral squamous cell carcinoma by targeting the miR-137-3p/FOXC1 axis and is associated with survival in the TCGA dataset ${ }^{59-61}$. The high expression of KLHDC7B-DT aggravates pancreatic ductal adenocarcinoma development via inducing cross-talk between cancer cells and macrophages ${ }^{62}$. Immune-associated IncRNA AL133467.1, was identified as a predictivefactor with significant prognostic value in ovarian carcinoma, corresponding to long survival ${ }^{63}$.Similar to our study, Lai et al. reported that they had utilized AC004585.1 and two risk parameters (tumour subtype and TNM stage)as independent indicators to develop a novel prognostic model in breast cancer patients ${ }^{64}$. Studies reported that LncRNA MIR4435-2HG can promote hepatocellular carcinoma proliferation and metastasis through the miR-22-3p/YWHAZ axis ${ }^{65}$ and promote the progression of head and neck squamous cell carcinoma by regulating the miR-383-5p/RBM3 axis $^{66}$. Another study found that the downregulation of MR4435-2HG suppresses breast cancer progression via the Wnt/beta-catenin signaling pathway ${ }^{67}$.Additionally, we first identified other LncRNAs(AC087239.1,AC015802.3,GORABAS1,AC121247.1,LINC00702,TDRKH-AS1,SIDT1-AS1,AC012213.3,HSD11B1-AS1,AL645608.7 and LRRC8C-DT囚as independent prognostic factors in BC patients. The risk model in the present study consists of 21 hypoxia-related genes representing a convenient detection tool in the clinic.

Recently, immune checkpoint inhibitors(ICls) have attracted attention in cancer immunotherapy. Tumours can influence immune checkpoint networks to protect themselves from the immune system. However, few studies have explored the relationship between ICls and tumour hypoxia. It has been reported that hypoxia can affect immune checkpoints, causing an up-regulation of PD-L1 and increased expression of $\mathrm{CTLA}^{68}$. Our results document higher expression of hypoxia-related biomarkers (eg.PDCD1, CD276, CD274) among patients in the low-risk group. Interestingly, in our further immunotherapy analysis, the low-risk group received higher scores in all four types of immunophenoscore. This may indicate that patients with low-risk scores are more suitable candidates for immunotherapy. 
In this study, we have established for the first time a model to develop and validate a hypoxia risk score based on 21 hypoxia-associated genes. This model served as an independent prognostic factor for BC patients and reflected the overall intensity of the immune response in the $\mathrm{BC}$ microenvironment. In summary, we developed and validated a hypoxia risk score model based on a novel hypoxia-related gene signature revealing the relationship between hypoxia and the tumour immune microenvironment. Nevertheless, further investigations in a larger cohort of patients are needed to verify the accuracy of our model.

\section{Conclusion}

In conclusion, our study constructed a novel prognostic model to predict the outcomes of BC patients and provide indications for novel targets for the management of BC.

\section{Methods}

\section{Data collection}

RNA-sequence data of 1208 individuals were extracted from the TCGA-BRCA database (112 normal and 1096 tumours). In this study, we downloaded the hypoxia-related gene set from Gene Set Enrichment (hallmark-hypoxia), a web-based consortium with data on 200 genes upregulated in response to low oxygen levels ${ }^{35}$.Overall, we identified 200 hypoxia-related genes (Table S1).Using a screening approach based on Pearson's correlation analysis (threshold of $|R 2|>0.4 ; P<0.001$ ), we were able to identify 274 hypoxia-related IncRNAs. The clinical information on the breast cancer patients such as gender, age, stage, grade, TMN, survival time and survival status were also accessed. The criterion of $|\log 2 \mathrm{FC}| \geq 1$, FDR $<0.05$ was used to select the significantly differentially-expressed hypoxia-related IncRNAs. First, we separately analyzed upregulated and downregulated genes among hypoxia-related differentiallyexpressed genes (DEGs). Then, Gene Ontology (GO) enrichment analysis was used to explore the biological pathways associated with the DEGs.Moreover, the biological processes (BP), cellular components (CC) and molecular functions (MF) were analyzed utilizing the Kyoto Encyclopedia of Genes and Genomes (KEGG) database using R software, ggplot2 package.The details are described in Figure 1.

\section{Development of the hypoxia-related IncRNA prognostic signature}

Hypoxia-related genes were extracted from the Molecular Signature Database (MSigDB, http://www.gseamsigdb.org/). We used univariate and multivariate Cox regression analyses to construct the hypoxiarelated IncRNA signature, stratified based on risk score (Coefficient IncRNA1 $\times$ expression of IncRNA1) + (Coefficient IncRNA2 $\times$ expression of IncRNA2) $+\cdots+$ (Coefficient IncRNA $\times$ expression IncRNA). The risk score was calculated for each sample, which were then divided into a high-risk and a low-risk group according to whether they were above or below the median.

\section{The predictive nomogram}


Gene set enrichment analyses (GSEA) were performed using the GSEA software to define the IncRNA signatures, which we then searched for in the TCGA-BRCA project. $\mathrm{P}<0.05$ and false discovery rate (FDR) q-value $<0.25$ were considered statistically significant. The nomogram was then constructed based on the results of multiple survival analyses according to the method described previously for predicting 1,3 , and 5-year OS of BC patients.

\section{Immunity analysis and gene expression}

We used currently accepted methods, including TIMER ${ }^{36,37}$, CIBERSORT $^{36,37}$, XCELL $^{38,39}$, QUANTISEQ ${ }^{40,41}$, MCPcounter ${ }^{42}$ and EPIC ${ }^{43}$, to assess cellular components or cellular immune responses and to compare high risk and low-risk groups based on hypoxia-related IncRNA signatures. A heatmap display was used to depict differences in the immune response through different algorithms. Apart from identifying immune function,ssGSEA was used to quantify the tumour-infiltrating immune cell subgroups. Potential immune checkpoints were also compared between high risk and low-risk groups.

\section{Clinical significance of the Risk Score model for treatment of invasive breast cancer}

We adopted the IC50 value for the chemotherapeutic drugs commonly used in the TCGA project to evaluate the model for the clinical treatment of BC. According to AJCC guidelines, chemotherapeutic drugs such as doxorubicin, docetaxel, paclitaxel, cisplatin, vinorelbine, gemcitabine, lapatinib and palbociclib were used for treating the BC patients. Differences in IC50 between high-risk and low-risk groups were compared by the Wilcoxon signed-rank test and R's pRRophetic and ggplot2 were used to construct a block diagram.

\section{Statistical analysis}

Bioconductor packages in R software(version 4.1.0)were used for data analysis. Normally distributed variables were analyzed using the unpaired student's t-test and non-normally distributed values were analyzed using the Wilcoxon test. The differently expressed IncRNAs were identified by a BenjaminiHochberg-based algorithm. GSVA, an R-package, was used to compare the ssGSEA-normalized BRCA DEGs with a genome. Decision curve analysis (DCA) was carried out and receiver operating characteristic (ROC) curves were constructed to evaluate the clinical fitness and accuracy of the nomogram, respectively ${ }^{44}$.Logistic regression analysis was used to evaluate the relationship between hypoxia-related IncRNAs and clinicopathological manifestations by heatmapping.Survival of BC patients based on the hypoxia-related IncRNA signatures was assessed using the Kaplan-Meier method. The criterion for each analysis for statistical significance was set at Pख0.05.

\section{Abbreviations}




\begin{tabular}{|c|c|}
\hline $\mathrm{BC}$ & Breast cancer \\
\hline TCGA & The Cancer Genome Atlas \\
\hline IncRNAs & Long Non-Coding RNAs \\
\hline AUC & ROC-curve \\
\hline GSEA & Gene set enrichment analysis \\
\hline APC & activated protein $\mathrm{C}$ \\
\hline CCR & Cinnamoyl CoA reductase \\
\hline MHC & major histocompatibility complex \\
\hline IFN & Interferons \\
\hline IC50 & half-maximal inhibitory concentration \\
\hline PFS & progression-free \\
\hline OS & overall survival \\
\hline EMT & epithelial-mesenchymal transition \\
\hline BP & Biological Process \\
\hline MF & Molecular Function \\
\hline $\mathrm{CC}$ & Cellular Component \\
\hline KEGG & Kyoto Encyclopedia of Genes and Genomes \\
\hline HIF & hypoxia-inducible factor \\
\hline MCP-Counter & Microenvironment Cell Populations-Counter method \\
\hline ssGSEA) ; & single-sample gene set enrichment analysis \\
\hline TIMER & Tumor Immunoassay Resource \\
\hline IPS & immunopheno-score \\
\hline EMT & epithelial-mesenchymal transition \\
\hline ICls & immune checkpoint inhibitors \\
\hline ssGSEA) ; & single-sample gene set enrichment analysis \\
\hline TIMER & Tumor Immunoassay Resource \\
\hline IPS & immunopheno-score \\
\hline EMT & epithelial-mesenchymal transition \\
\hline ICls & immune checkpoint inhibitors \\
\hline
\end{tabular}




\section{Declarations}

\section{Acknowledgements}

We thank Shuang Shen for help with some of the statistical analyses. We would like to express their gratitude to EditSprings (https://www.editsprings.cn/) for the expert linguistic services provided.

\section{Funding}

The work was supported by Clinical Project Foundation from Department of Science and Technology of Guizhou Province( LC[2021]004), the General Project Foundation of Science and Technology Bureau of Zunyi City(HZ (2019) 133,HZ (2019) 175).

\section{Availability of data and materials}

All data generated or analyzed during this study are included in this manuscript.

\section{Consent for Publication}

All authors consent for publication.

\section{Author contributions}

J.L.H, S.S., and C.C. designed the study, offered critical input, and co-wrote the manuscript. D.O.,R.Q conducted the experiments, wrote the manuscript, drew and prepared figures and analyzed the data. S.S.,Y.M.G analyzed samples using R software. D.O. supervised the study.

\section{Competing interests}

The authors declare no competing interests.

\section{Ethics approval and consent to participate}

Not applicable.

\section{References}

1 Sharma, R. Breast cancer incidence, mortality and mortality-to-incidence ratio (MIR) are associated with human development, 1990-2016: evidence from Global Burden of Disease Study 2016. Breast Cancer26, 428-445, doi:10.1007/s12282-018-00941-4 (2019). 
2 Lenga, L. et al. lodine Map Radiomics in Breast Cancer: Prediction of Metastatic Status. Cancers (Basel)13, doi:10.3390/cancers13102431 (2021).

3 Siegel, R. L., Miller, K. D. \& Jemal, A. Cancer statistics, 2019. CA Cancer J Clin69, 7-34, doi:10.3322/caac.21551 (2019).

4 Rodriguez, D. et al. The Central Contributions of Breast Cancer Stem Cells in Developing Resistance to Endocrine Therapy in Estrogen Receptor (ER)-Positive Breast Cancer. Cancers (Basel)11, doi:10.3390/cancers11071028 (2019).

5 Liu, K. \& Ma, R. MicroRNA-615-5p regulates the proliferation and apoptosis of breast cancer cells by targeting HSF1. Exp Ther Med21, 192, doi:10.3892/etm.2021.9624 (2021).

6 Valdora, F., Houssami, N., Rossi, F., Calabrese, M. \& Tagliafico, A. S. Rapid review: radiomics and breast cancer. Breast Cancer Res Treat169, 217-229, doi:10.1007/s10549-018-4675-4 (2018).

7 Yang, Y. et al. The diagnostic value of DNA repair gene in breast cancer metastasis. Sci Rep10, 19626, doi:10.1038/s41598-020-76577-2 (2020).

$8 \mathrm{Kim}, \mathrm{D}$. A. et al. Tannic acid attenuates the formation of cancer stem cells by inhibiting NF-kappaBmediated phenotype transition of breast cancer cells. Am J Cancer Res9, 1664-1681 (2019).

9 Zhang, Z. et al. Breast cancer metastasis suppressor OTUD1 deubiquitinates SMAD7. Nat Commun8, 2116, doi:10.1038/s41467-017-02029-7 (2017).

10 Darbeheshti, F. et al. Circular RNA hsa_circ_0044234 as distinct molecular signature of triple negative breast cancer: a potential regulator of GATA3. Cancer Cell Int21, 312, doi:10.1186/s12935-021-02015-6 (2021).

$11 \mathrm{Ma}, \mathrm{H} ., \mathrm{He}, \mathrm{Z}$., Chen, J., Zhang, X. \& Song, P. Identifying of biomarkers associated with gastric cancer based on 11 topological analysis methods of CytoHubba. Sci Rep11, 1331, doi:10.1038/s41598-02079235-9 (2021).

12 Petrova, V., Annicchiarico-Petruzzelli, M., Melino, G. \& Amelio, I. The hypoxic tumour microenvironment. Oncogenesis7, 10, doi:10.1038/s41389-017-0011-9 (2018).

13 Surono, A., Priyanto, P. \& Indrasari, S. R. Hypoxia-Inducible Factor-1alpha Expression in Indonesian Laryngeal Squamous Cell Carcinoma Patients. J Onco/2016, 3215463, doi:10.1155/2016/3215463 (2016).

14 Metsala, O. et al. Transportable system enabling multiple irradiation studies under simultaneous hypoxia in vitro. Radiat Onco/13, 220, doi:10.1186/s13014-018-1169-9 (2018). 
15 Erler, J. T. et al. Hypoxia-mediated down-regulation of Bid and Bax in tumors occurs via hypoxiainducible factor 1-dependent and -independent mechanisms and contributes to drug resistance. Mol Cell Bio/24, 2875-2889, doi:10.1128/MCB.24.7.2875-2889.2004 (2004).

16 Muhleisen, A., Giaisi, M., Kohler, R., Krammer, P. H. \& Li-Weber, M. Tax contributes apoptosis resistance to HTLV-1-infected T cells via suppression of Bid and Bim expression. Cell Death Dis5, e1575, doi:10.1038/cddis.2014.536 (2014).

17 Omran, Z. et al. Design, Synthesis and In-Vitro Biological Evaluation of Antofine and Tylophorine Prodrugs as Hypoxia-Targeted Anticancer Agents. Molecules26, doi:10.3390/molecules26113327 (2021).

18 Pennacchietti, S. et al. Hypoxia promotes invasive growth by transcriptional activation of the met protooncogene. Cancer Cel/3, 347-361, doi:10.1016/s1535-6108(03)00085-0 (2003).

19 De Miguel, M. P., Alcaina, Y., de la Maza, D. S. \& Lopez-Iglesias, P. Cell metabolism under microenvironmental low oxygen tension levels in stemness, proliferation and pluripotency. Curr Mol Med15, 343-359, doi:10.2174/1566524015666150505160406 (2015).

20 Al Tameemi, W., Dale, T. P., Al-Jumaily, R. M. K. \& Forsyth, N. R. Hypoxia-Modified Cancer Cell Metabolism. Front Cell Dev Bio/7, 4, doi:10.3389/fcell.2019.00004 (2019).

21 Jongen, J. M. J. et al. Downregulation of DNA repair proteins and increased DNA damage in hypoxic colon cancer cells is a therapeutically exploitable vulnerability. Oncotarget8, 86296-86311, doi:10.18632/oncotarget.21145 (2017).

22 Glazer, P. M., Hegan, D. C., Lu, Y., Czochor, J. \& Scanlon, S. E. Hypoxia and DNA repair. Yale J Biol Med86, 443-451 (2013).

23 Kaplan, A. R. \& Glazer, P. M. Impact of hypoxia on DNA repair and genome integrity. Mutagenesis35, 61-68, doi:10.1093/mutage/gez019 (2020).

24 Bronisz, A., Salinska, E., Chiocca, E. A. \& Godlewski, J. Hypoxic Roadmap of Glioblastoma-Learning about Directions and Distances in the Brain Tumor Environment. Cancers (Basel)12, doi:10.3390/cancers12051213 (2020).

25 Wang, Z. et al. Cardamonin exerts anti-gastric cancer activity via inhibiting LncRNA-PVT1-STAT3 axis. Biosci Rep39, doi:10.1042/BSR20190357 (2019).

26 Xiong, T. et al. LncRNA NRON promotes the proliferation, metastasis and EMT process in bladder cancer. Journal of Cancer11, 1751-1760, doi:10.7150/jca.37958 (2020).

27 Li, W. et al. Long Non-Coding RNA (LncRNA) HOXA11-AS Promotes Breast Cancer Invasion and Metastasis by Regulating Epithelial-Mesenchymal Transition. Medical science monitor : international medical journal of experimental and clinical research23, 3393-3403, doi:10.12659/msm.904892 (2017). 
28 Yang, Y. X. et al. Long non-coding RNA p10247, high expressed in breast cancer (IncRNA-BCHE), is correlated with metastasis. Clin Exp Metastasis35, 109-121, doi:10.1007/s10585-018-9901-2 (2018).

29 Sun, Y. et al. A five IncRNA signature for prognosis prediction in hepatocellular carcinoma. Mol Med Rep19, 5237-5250, doi:10.3892/mmr.2019.10203 (2019).

30 Zhang, Y. et al. LASSObased CoxPH model identifies an 11 IncRNA signature for prognosis prediction in gastric cancer. Mol Med Rep18, 5579-5593, doi:10.3892/mmr.2018.9567 (2018).

31 Zhao, Q. J., Zhang, J., Xu, L. \& Liu, F. F. Identification of a five-long non-coding RNA signature to improve the prognosis prediction for patients with hepatocellular carcinoma. World J Gastroentero/24, 3426-3439, doi:10.3748/wjg.v24.i30.3426 (2018).

32 Zhang, H., Qin, C., Liu, H. W., Guo, X. \& Gan, H. An Effective Hypoxia-Related Long Non-Coding RNAs Assessment Model for Prognosis of Clear Cell Renal Carcinoma. Front Onco/11, 616722, doi:10.3389/fonc.2021.616722 (2021).

33 Chen, Q., Hu, L. \& Chen, K. Construction of a Nomogram Based on a Hypoxia-Related IncRNA Signature to Improve the Prediction of Gastric Cancer Prognosis. Front Genet11, 570325, doi:10.3389/fgene.2020.570325 (2020).

34 Gong, P. J. et al. Hypoxia-Associated Prognostic Markers and Competing Endogenous RNA CoExpression Networks in Breast Cancer. Front Onco/10, 579868, doi:10.3389/fonc.2020.579868 (2020).

$35 \mathrm{Li}, \mathrm{R}$. et al. Hypoxia pathway genetic variants predict survival of non-small-cell lung cancer patients receiving platinum-based chemotherapy. Carcinogenesis38, 419-424, doi:10.1093/carcin/bgx014 (2017).

36 Charoentong, P. et al. Pan-cancer Immunogenomic Analyses Reveal Genotype-Immunophenotype Relationships and Predictors of Response to Checkpoint Blockade. Cell Rep18, 248-262, doi:10.1016/j.celrep.2016.12.019 (2017).

37 Newman, A. M. et al. Robust enumeration of cell subsets from tissue expression profiles. Nat Methods12, 453-457, doi:10.1038/nmeth.3337 (2015).

38 Aran, D. Cell-Type Enrichment Analysis of Bulk Transcriptomes Using xCell. Methods Mol Bio/2120, 263-276, doi:10.1007/978-1-0716-0327-7_19 (2020).

39 Aran, D., Hu, Z. \& Butte, A. J. xCell: digitally portraying the tissue cellular heterogeneity landscape. Genome Bio/18, 220, doi:10.1186/s13059-017-1349-1 (2017).

40 Plattner, C., Finotello, F. \& Rieder, D. Deconvoluting tumor-infiltrating immune cells from RNA-seq data using quanTIseq. Methods Enzymo/636, 261-285, doi:10.1016/bs.mie.2019.05.056 (2020). 
41 Finotello, F. et al. Molecular and pharmacological modulators of the tumor immune contexture revealed by deconvolution of RNA-seq data. Genome Med11, 34, doi:10.1186/s13073-019-0638-6 (2019).

$42 \mathrm{Shi}$, J. et al. LPAR1, Correlated With Immune Infiltrates, Is a Potential Prognostic Biomarker in Prostate Cancer. Front Onco/10, 846, doi:10.3389/fonc.2020.00846 (2020).

43 Racle, J., de Jonge, K., Baumgaertner, P., Speiser, D. E. \& Gfeller, D. Simultaneous enumeration of cancer and immune cell types from bulk tumor gene expression data. Elife6, doi:10.7554/eLife.26476 (2017).

$44 \mathrm{Mao}, \mathrm{C}$. et al. Concordance Between Watson for Oncology and Multidisciplinary Teams in Colorectal Cancer: Prognostic Implications and Predicting Concordance. Front Onco/10, 595565, doi:10.3389/fonc.2020.595565 (2020).

45 Zhang, X. et al. Hypoxic BMSC-derived exosomal miRNAs promote metastasis of lung cancer cells via STAT3-induced EMT. Mol Cancer18, 40, doi:10.1186/s12943-019-0959-5 (2019).

46 Schito, L. Hypoxia-Dependent Angiogenesis and Lymphangiogenesis in Cancer. Adv Exp Med Bio/1136, 71-85, doi:10.1007/978-3-030-12734-3_5 (2019).

47 Klaus, A., Fathi, O., Tatjana, T. W., Bruno, N. \& Oskar, K. Expression of Hypoxia-Associated Protein HIF1alpha in Follicular Thyroid Cancer is Associated with Distant Metastasis. Pathol Oncol Res24, 289-296, doi:10.1007/s12253-017-0232-4 (2018).

48 Chakhoyan, A. et al. FMISO-PET-derived brain oxygen tension maps: application to glioblastoma and less aggressive gliomas. Sci Rep7, 10210, doi:10.1038/s41598-017-08646-y (2017).

49 Guo, Y. et al. Hypoxiainducible factors in hepatocellular carcinoma (Review). Oncol Rep43, 3-15, doi:10.3892/or.2019.7397 (2020).

50 Wing, P. A. C. et al. Hypoxia inducible factors regulate hepatitis B virus replication by activating the basal core promoter. J Hepato/75, 64-73, doi:10.1016/j.jhep.2020.12.034 (2021).

51 Tan, Y. E. et al. LINC01235-TWIST2 feedback loop facilitates epithelial-mesenchymal transition in gastric cancer by inhibiting THBS2. Aging (Albany NY)12, 25060-25075, doi:10.18632/aging.103979 (2020).

52 Ding, Y. et al. A Novel Autophagy-Related IncRNA Gene Signature to Improve the Prognosis of Patients with Melanoma. Biomed Res Int2021, 8848227, doi:10.1155/2021/8848227 (2021).

53 Wan, J. et al. Autophagy-Related Long Non-coding RNA Is a Prognostic Indicator for Bladder Cancer. Front Onco/11, 647236, doi:10.3389/fonc.2021.647236 (2021). 
54 Gao, M. et al. A Six-IncRNA Signature for Immunophenotype Prediction of Glioblastoma Multiforme. Front Genet11, 604655, doi:10.3389/fgene.2020.604655 (2020).

55 Chen, P. et al. A Prognostic Model Based on Immune-Related Long Non-Coding RNAs for Patients With Cervical Cancer. Front Pharmaco/11, 585255, doi:10.3389/fphar.2020.585255 (2020).

56 Wang, D., Zhang, H., Fang, X., Cao, D. \& Liu, H. Pan-cancer analysis reveals the role of long non-coding RNA LINC01614 as a highly cancer-dependent oncogene and biomarker. Oncol Lett20, 1383-1399, doi:10.3892/ol.2020.11648 (2020).

57 Zhang, X. et al. Long non-coding RNA LINC02446 suppresses the proliferation and metastasis of bladder cancer cells by binding with EIF3G and regulating the mTOR signalling pathway. Cancer Gene Ther, doi:10.1038/s41417-020-00285-2 (2021).

58 Zhou, B., Guo, H. \& Tang, J. Long Non-Coding RNA TFAP2A-AS1 Inhibits Cell Proliferation and Invasion in Breast Cancer via miR-933/SMAD2. Medical science monitor : international medical journal of experimental and clinical research25, 1242-1253, doi:10.12659/MSM.912421 (2019).

59 Acha-Sagredo, A. et al. Long non-coding RNA dysregulation is a frequent event in non-small cell lung carcinoma pathogenesis. Br J Cancer122, 1050-1058, doi:10.1038/s41416-020-0742-9 (2020).

60 Ye, J., Wu, S., Pan, S., Huang, J. \& Ge, L. Risk scoring based on expression of long noncoding RNAs can effectively predict survival in hepatocellular carcinoma patients with or without fibrosis. Oncol Rep43, 1451-1466, doi:10.3892/or.2020.7528 (2020).

61 Che, H., Che, Y., Zhang, Z. \& Lu, Q. Long Non-Coding RNA LINC01929 Accelerates Progression of Oral Squamous Cell Carcinoma by Targeting the miR-137-3p/FOXC1 Axis. Front Onco/11, 657876, doi:10.3389/fonc.2021.657876 (2021).

$62 \mathrm{Li}, \mathrm{M}$. X. et al. KLHDC7B-DT aggravates pancreatic ductal adenocarcinoma development via inducing cross-talk between cancer cells and macrophages. Clin Sci (Lond)135, 629-649, doi:10.1042/CS20201259 (2021).

63 Li, Y., Huo, F. F., Wen, Y. Y. \& Jiang, M. Screening and Identification of an Immune-Associated IncRNA Prognostic Signature in Ovarian Carcinoma: Evidence from Bioinformatic Analysis. Biomed Res Int2021, 6680036, doi:10.1155/2021/6680036 (2021).

$64 \mathrm{Lai}, \mathrm{J}$. et al. Molecular characterization of breast cancer: a potential novel immune-related IncRNAs signature. J Trans/ Med18, 416, doi:10.1186/s12967-020-02578-4 (2020).

65 Shen, X., Ding, Y., Lu, F., Yuan, H. \& Luan, W. Long noncoding RNA MIR4435-2HG promotes hepatocellular carcinoma proliferation and metastasis through the miR-22-3p/YWHAZ axis. Am J TransI Res12, 6381-6394 (2020). 
66 Wang, S., Chen, X. \& Qiao, T. Long noncoding RNA MIR44352HG promotes the progression of head and neck squamous cell carcinoma by regulating the miR3835p/RBM3 axis. Oncol Rep45, doi:10.3892/or.2021.8050 (2021).

67 Chen, D. et al. Downregulation of long non-coding RNA MR4435-2HG suppresses breast cancer progression via the Wnt/beta-catenin signaling pathway. Oncol Lett21, 373, doi:10.3892/ol.2021.12634 (2021).

68 Horsman, M. R., Wittenborn, T. R., Nielsen, P. S. \& Elming, P. B. Tumors Resistant to Checkpoint Inhibitors Can Become Sensitive after Treatment with Vascular Disrupting Agents. Int J Mol Sci21, doi:10.3390/ijms21134778 (2020).

\section{Figures}

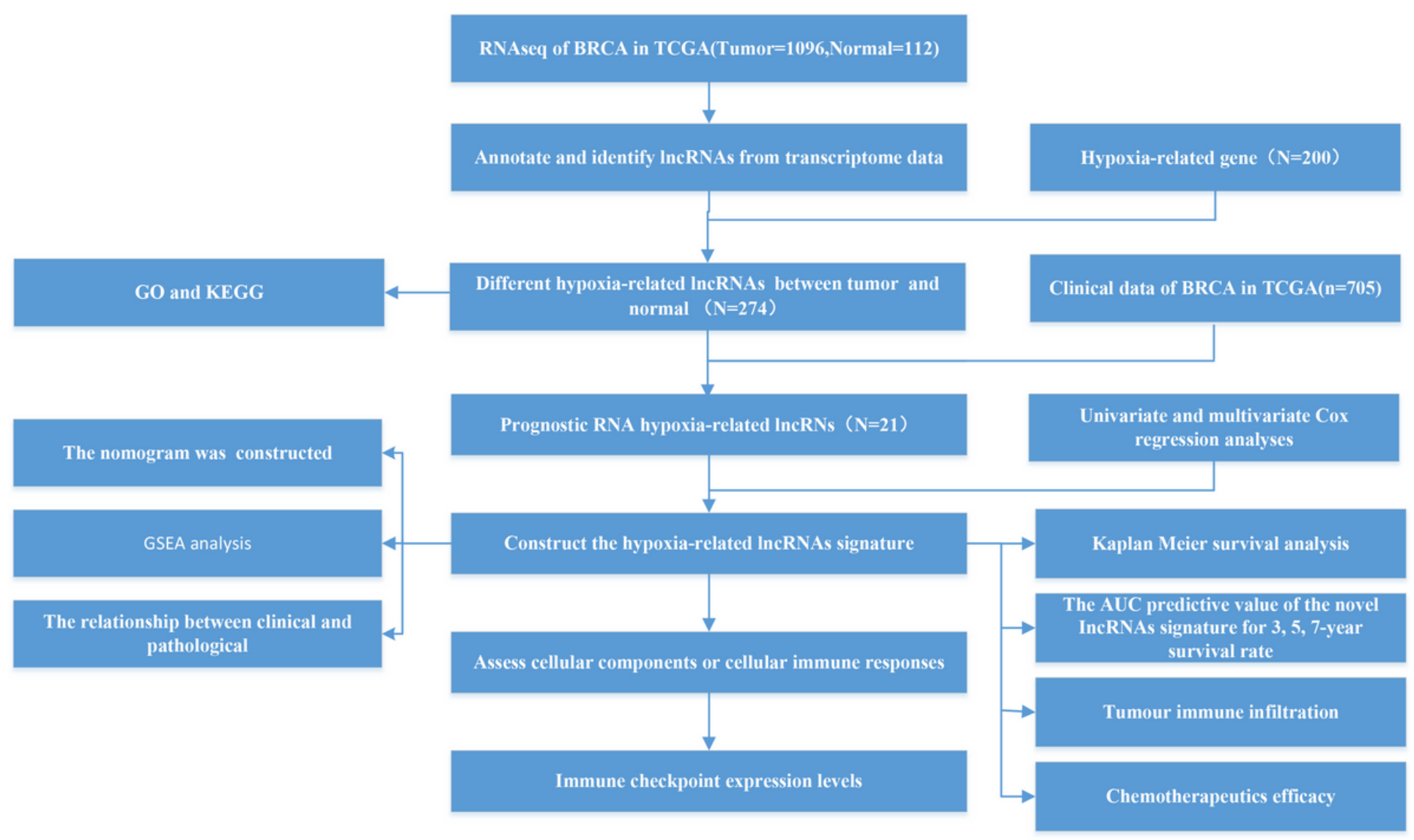

Figure 1

Flowchart for bioinformatics analysis of publicly available data from TCGA databases. 
A

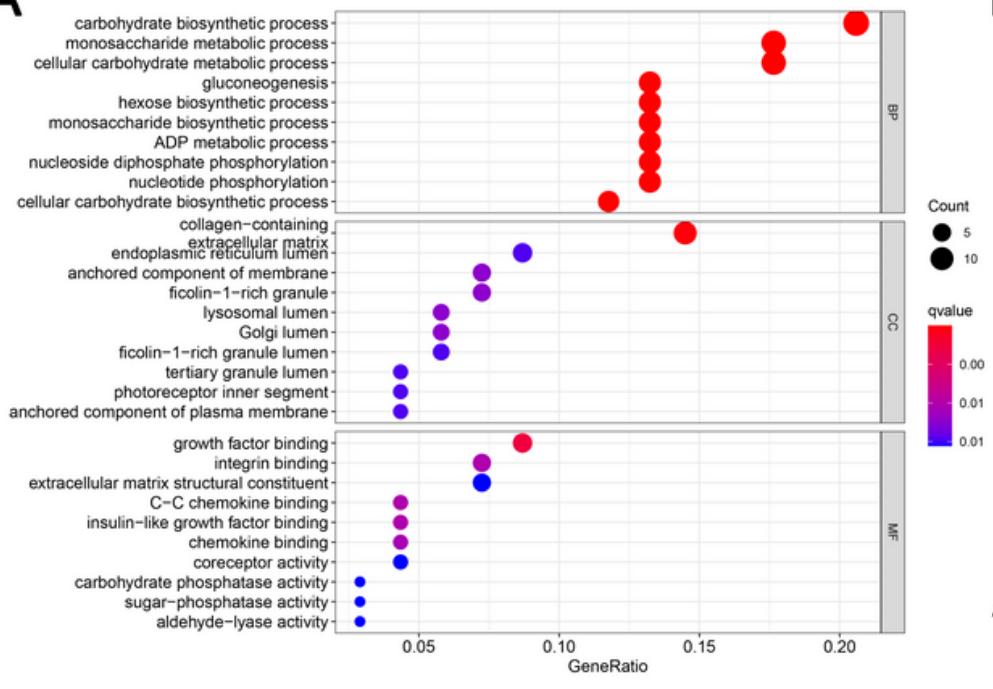

B

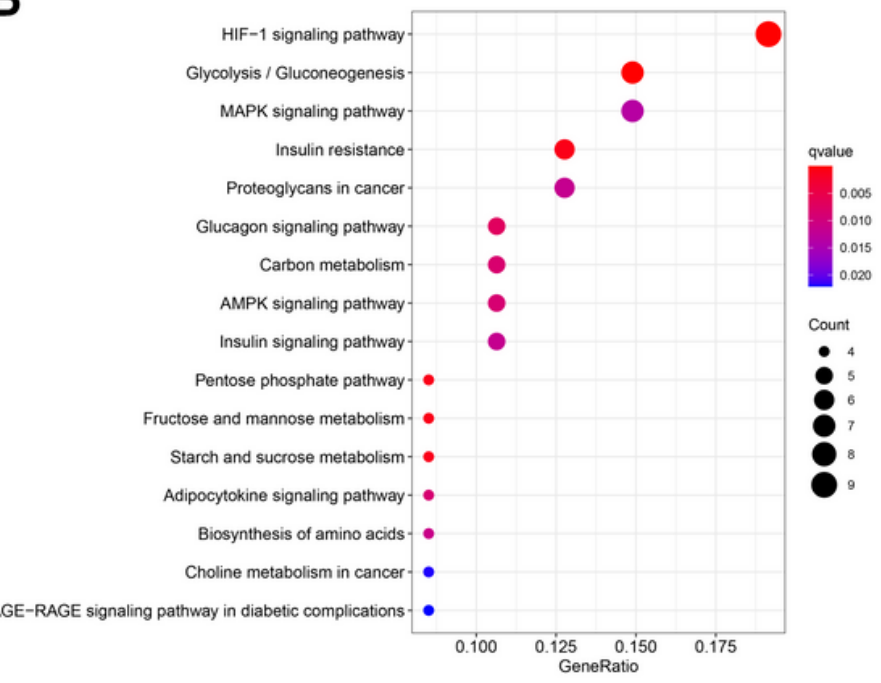

Figure 2

GO and KEGG analyses for hypoxia-related differentially expressed genes. (A) GO and (B) KEGG.
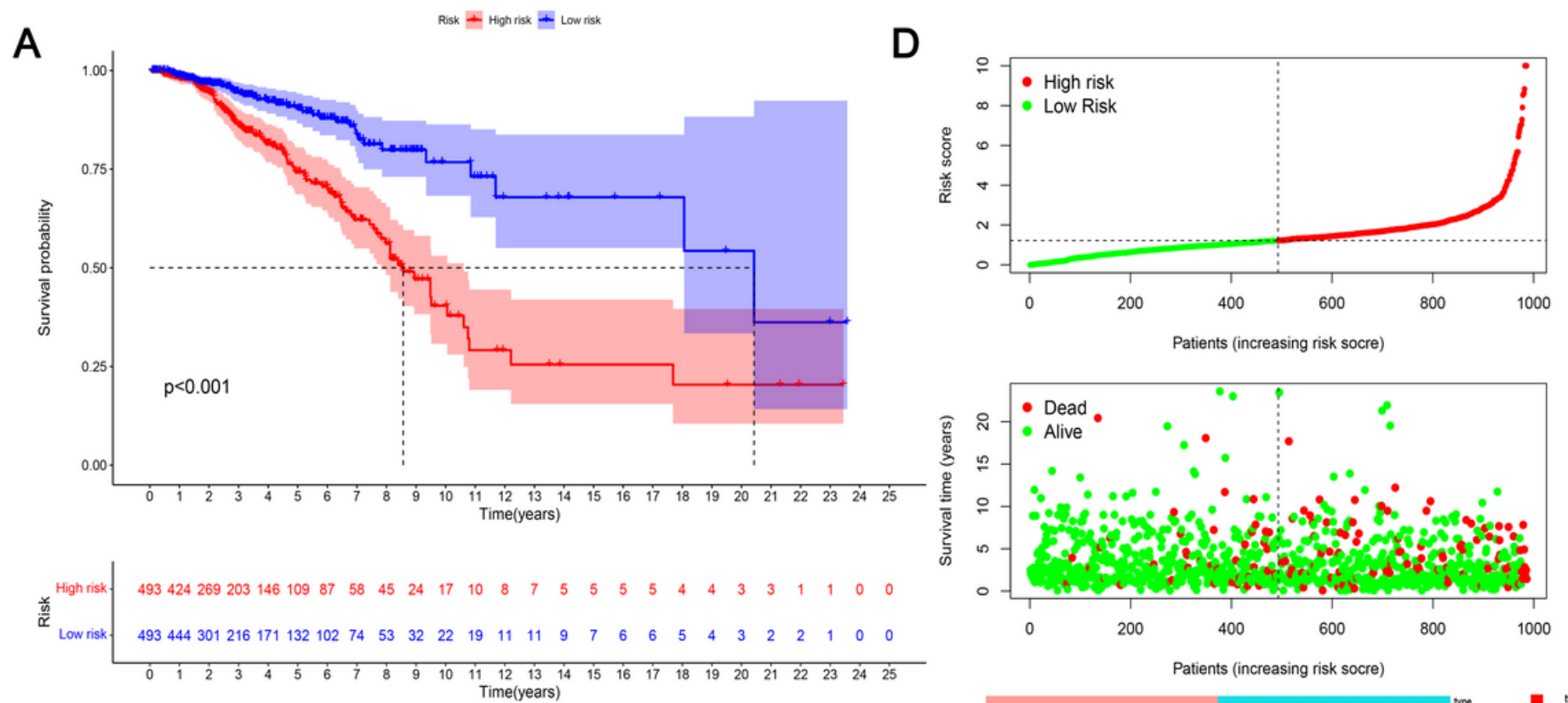

B

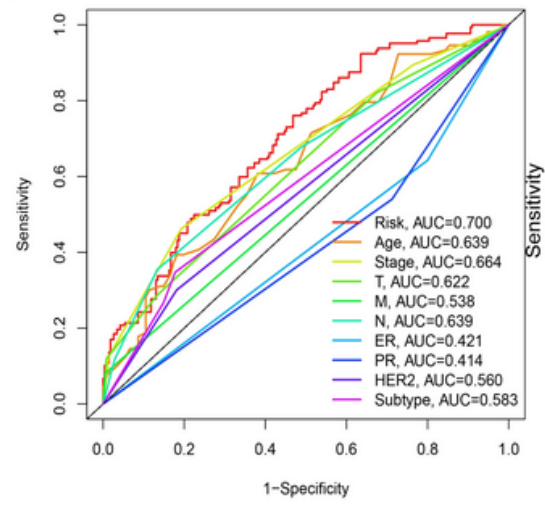

C

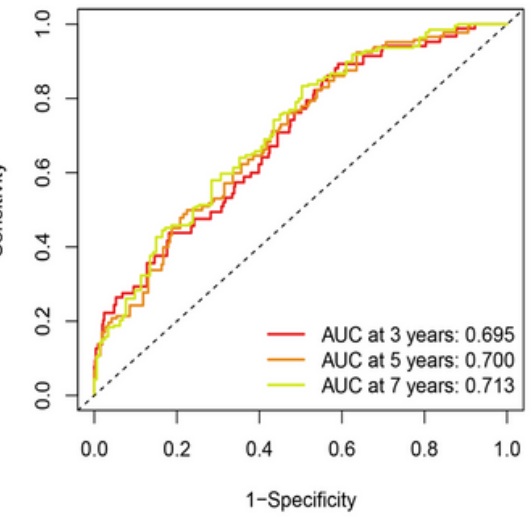

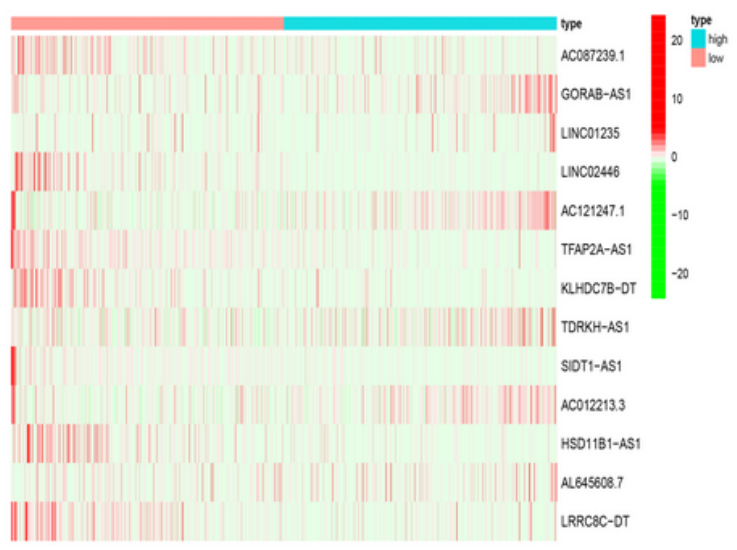




\section{Figure 3}

Hypoxia-related IncRNAs signature based on TCGA. (A) Kaplan-Meier curves result, (B). The AUC values of the risk factors, (C)The AUC of the for the prediction of 3, 5,7-year survival rate of BC and (D) Risk survival status plot.

A

$\begin{array}{lrr} & \text { pvalue } & \text { Hazard ratio } \\ \text { Age } & <0.001 & 1.041(1.022-1.060) \\ \text { Stage } & <0.001 & 2.341(1.712-3.202) \\ \mathrm{T} & <0.001 & 1.733(1.264-2.375) \\ \mathrm{M} & <0.001 & 9.734(4.822-19.647) \\ \mathrm{N} & <0.001 & 1.743(1.377-2.206) \\ \text { ER } & 0.079 & 0.639(0.388-1.053) \\ \text { PR } & 0.261 & 0.765(0.479-1.221) \\ \text { HER2 } & 0.143 & 1.490(0.874-2.538) \\ \text { Subtype } & 0.066 & 1.284(0.984-1.674) \\ \text { riskScore } & <0.001 & 1.169(1.116-1.223)\end{array}$

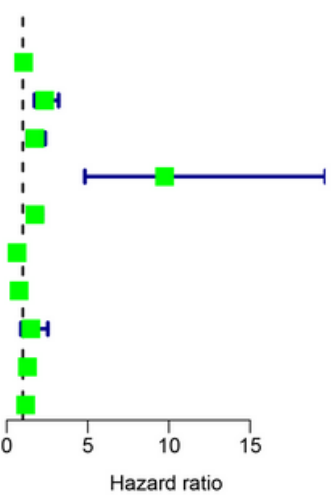

B

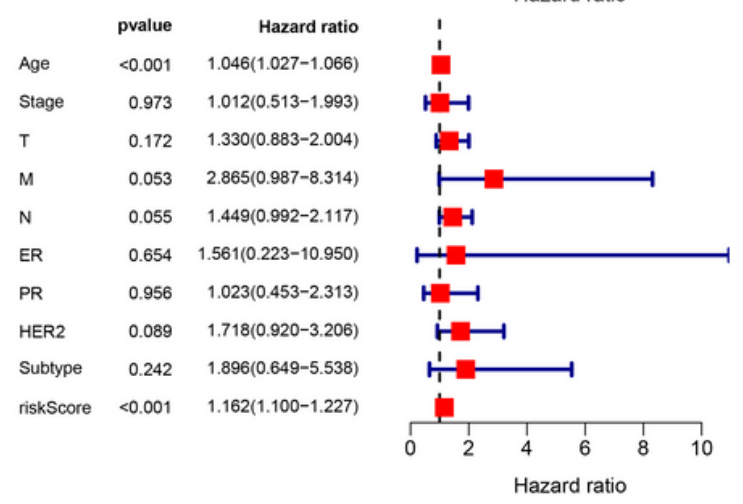

\section{C}

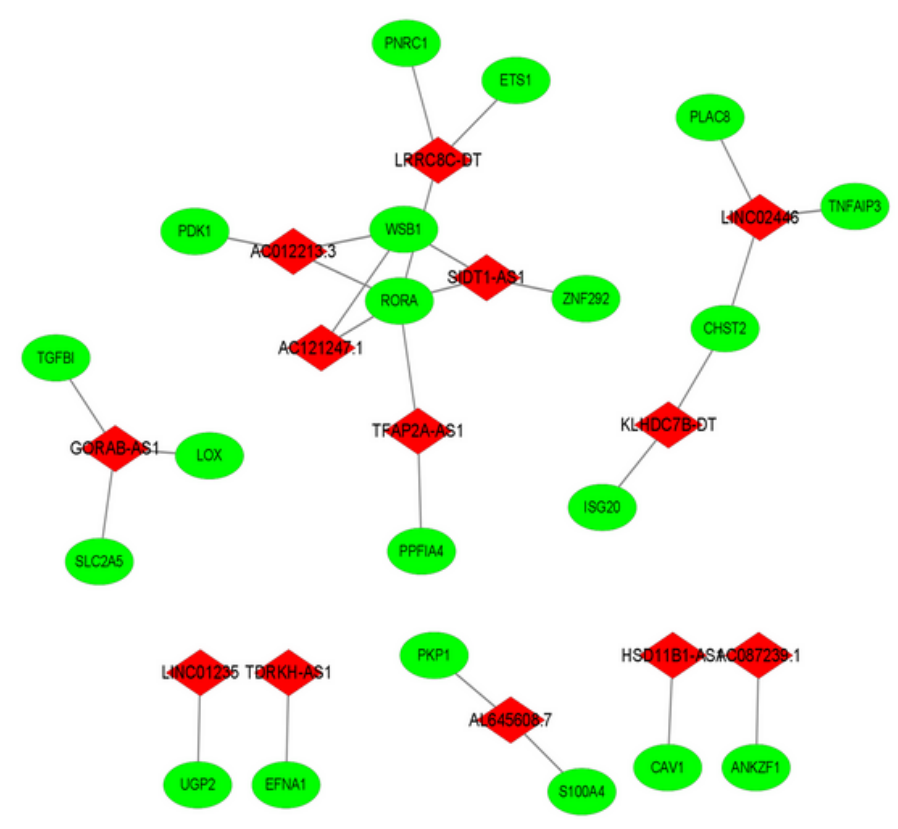

Figure 4

Univariate and multivariate COX analysis for the expression of Hypoxia-related IncRNAs. (A). univariate, (B). multivariate, (C). The relationship between thenovel IncRNA and mRNA expression. 


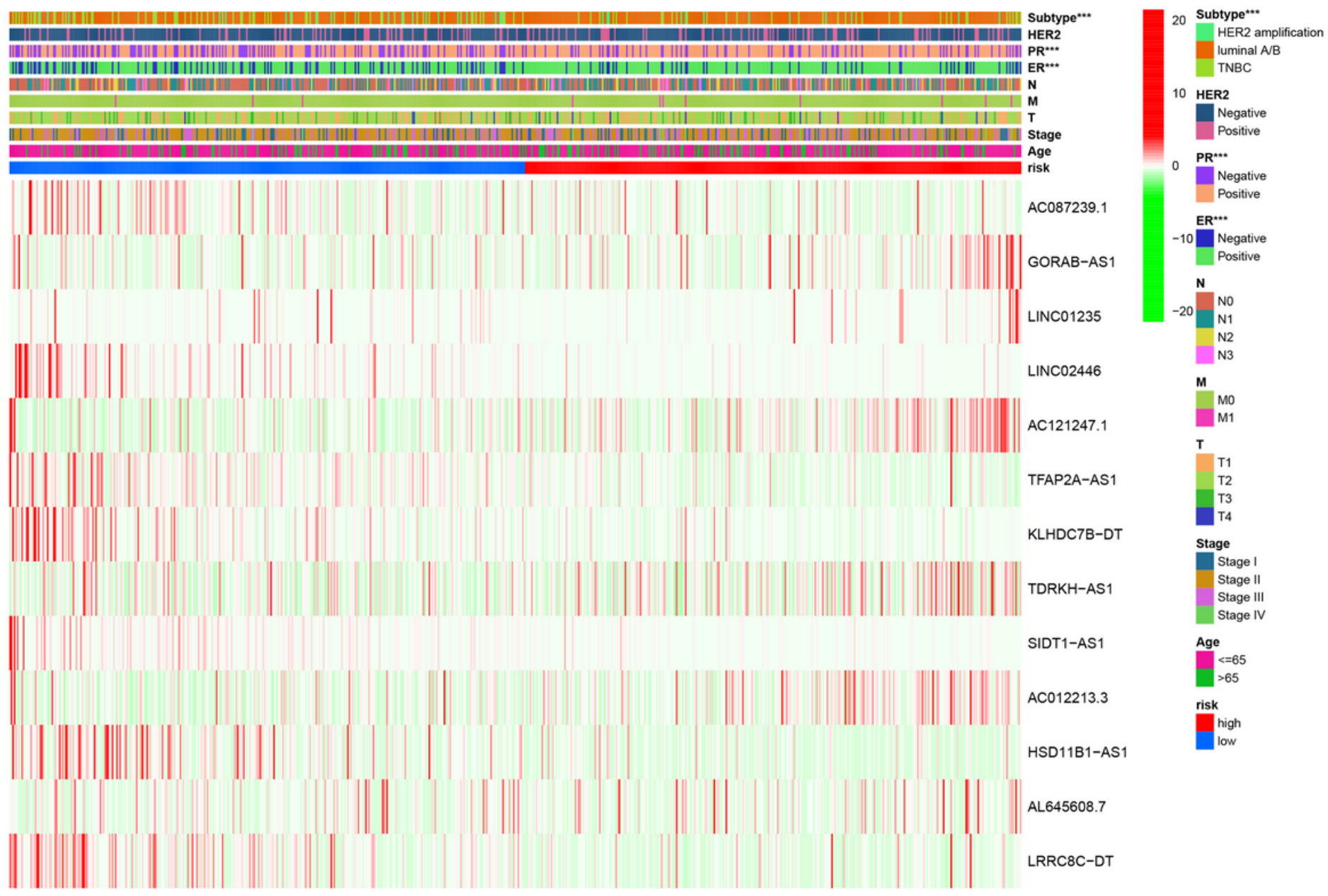

Figure 5

Heatmap for ferroptosis-related IncRNAs prognostic signature and clinicopathological manifestations. 


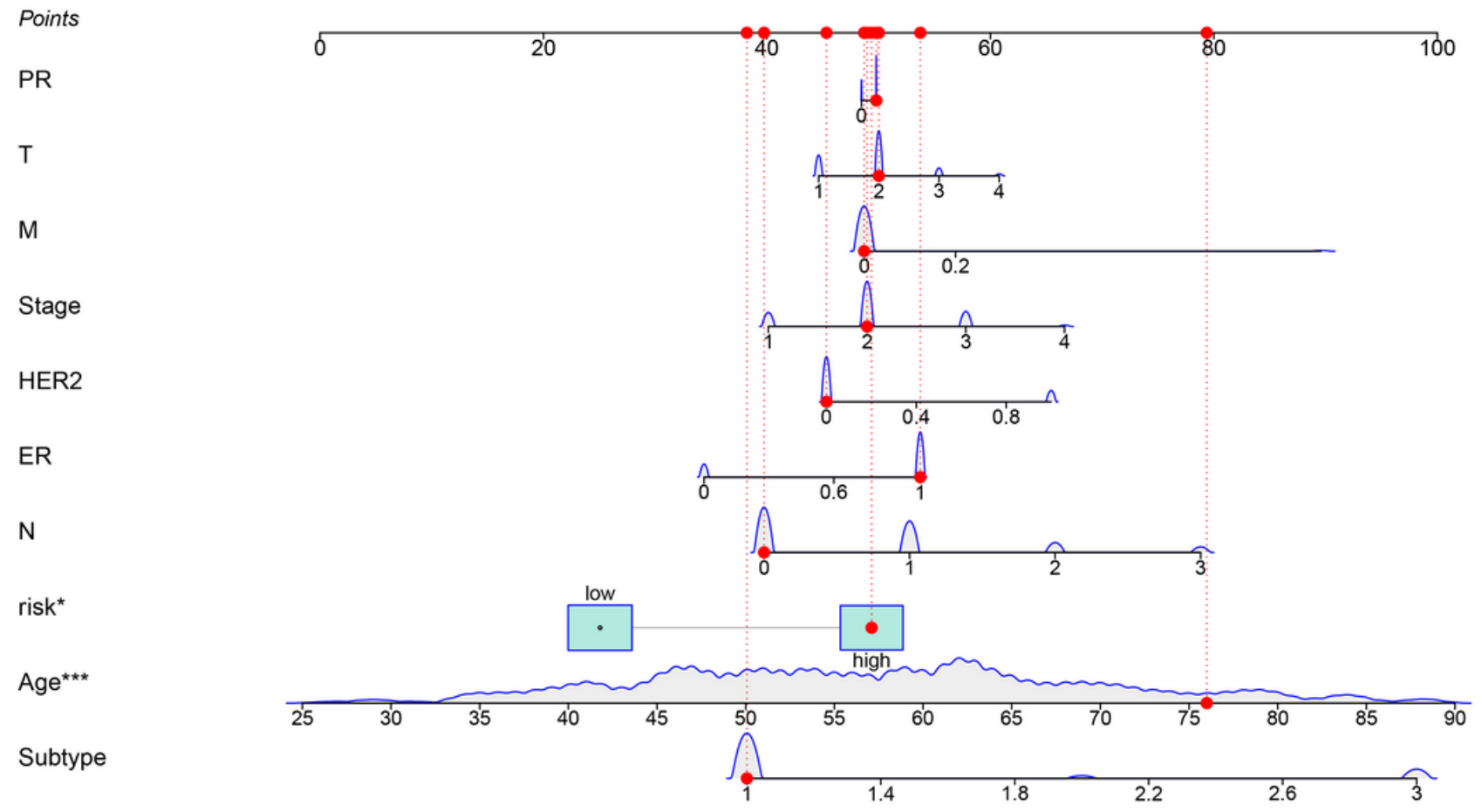

Total points

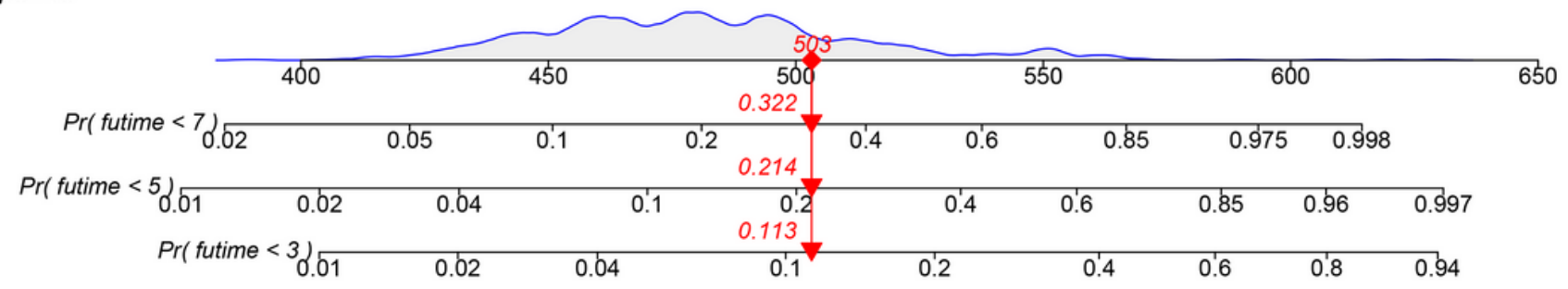

\section{Figure 6}

A nomogram for both clinic-pathological factors and prognostic hypoxia-related IncRNAs.

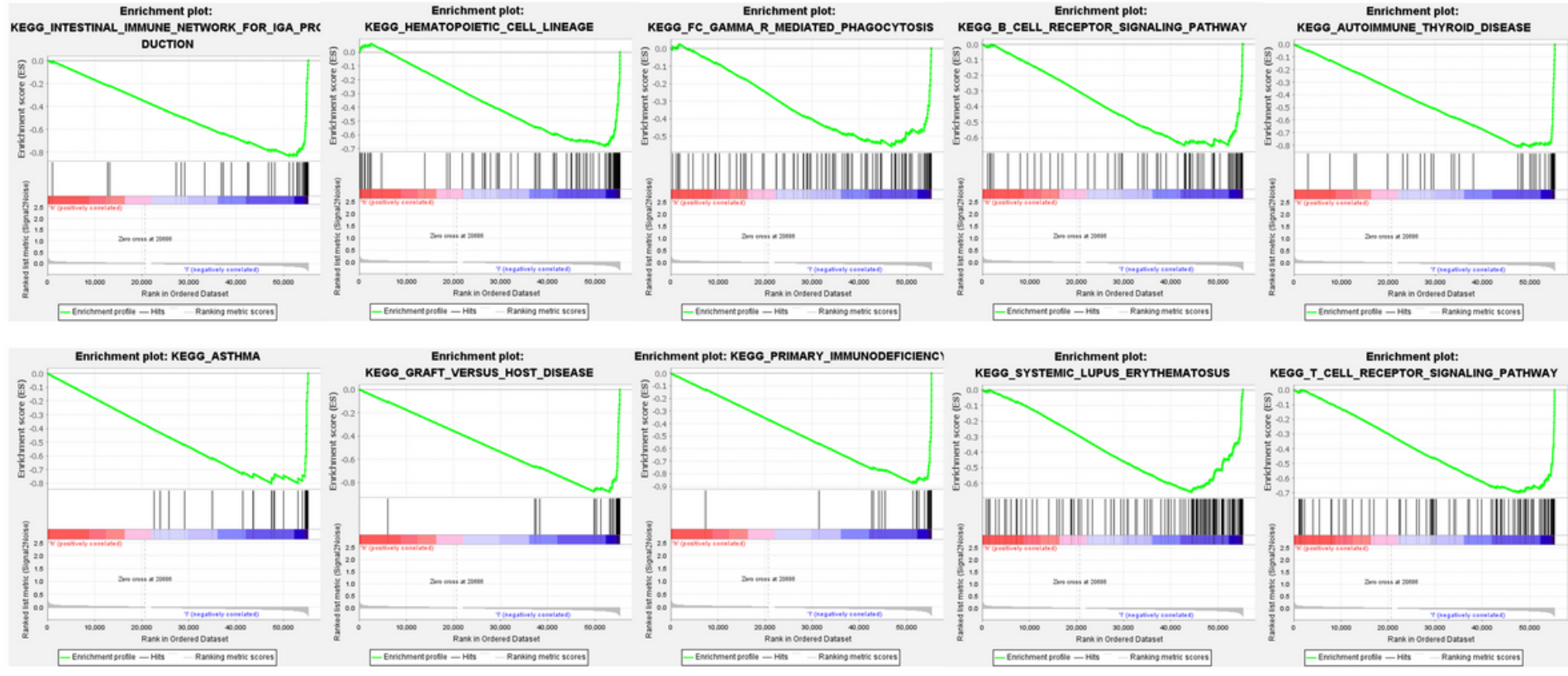


Figure 7

Gene set enrichment analysis (GSEA) enrichment map for hypoxia-related IncRNAs.
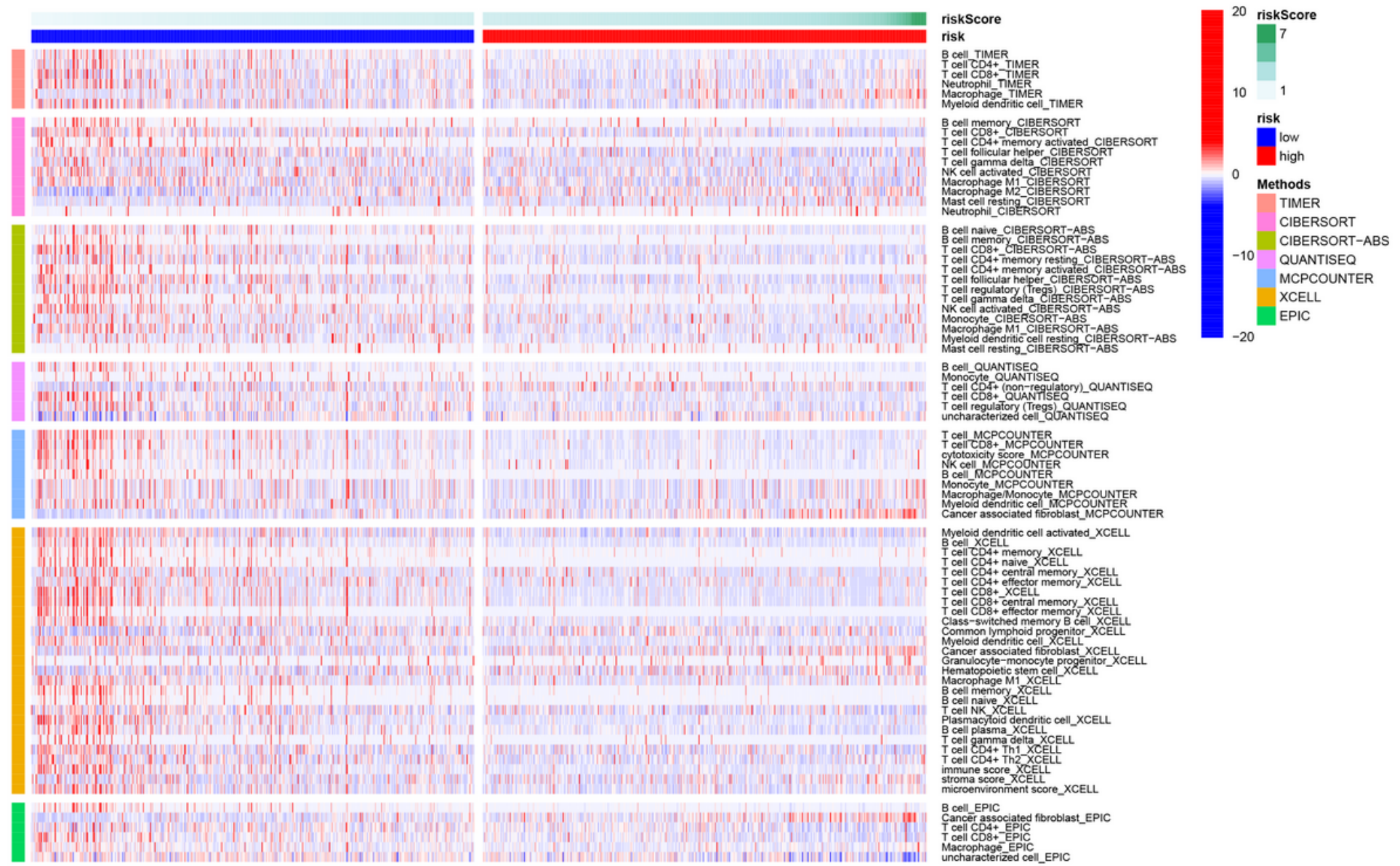

항

Figure 8

Heatmap for immune responses among high and low risk groups. 
A

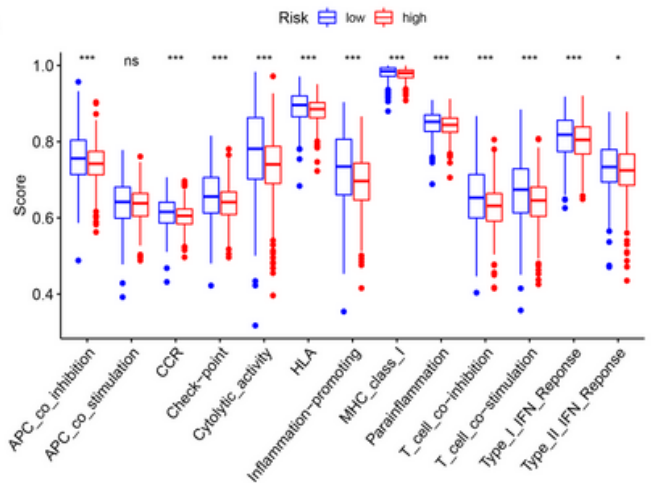

B Risk 追 low 早 high

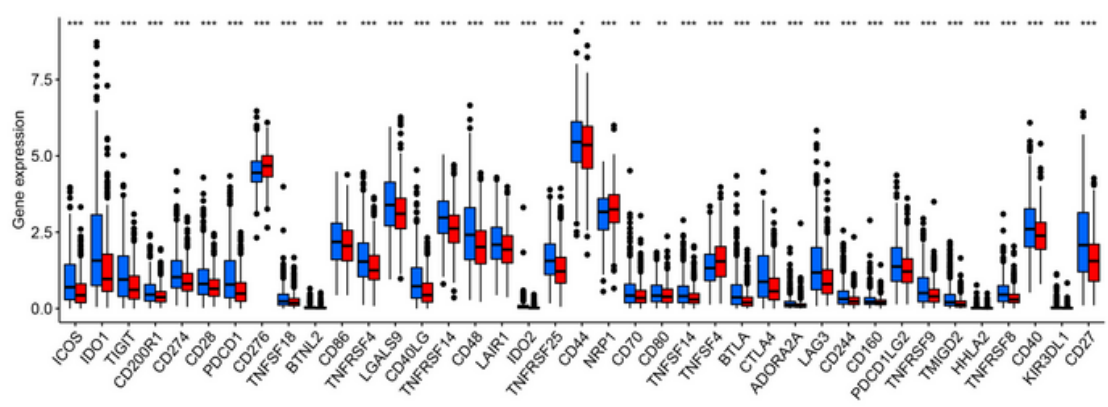

C
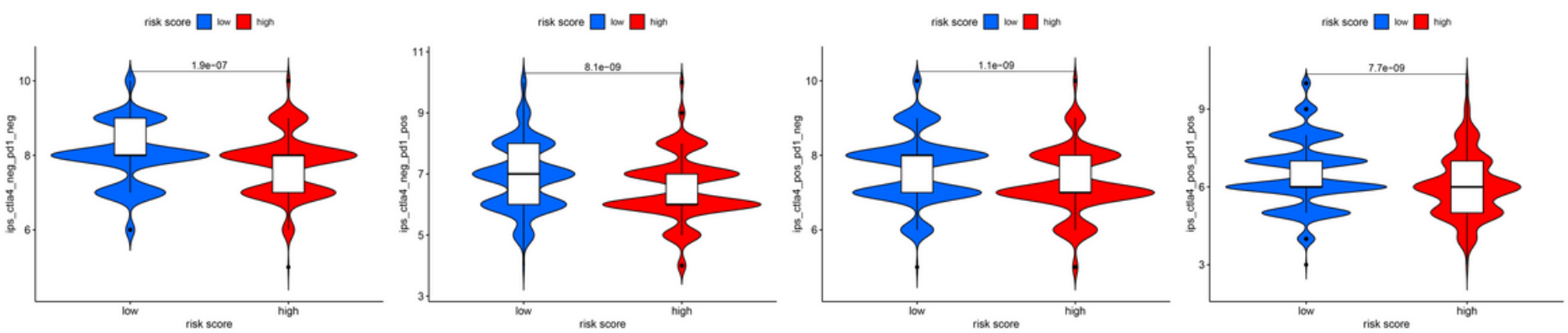

Figure 9

(A). Correlation analysis between immune-related IncRNAs and immune cell subpopulations.(B). Expression of immune checkpoints amonghigh and low risk groups.(C).Immunophenogram analysis to investigate the correlation between the immunopheno-score (IPS) and low/high risk groups. 


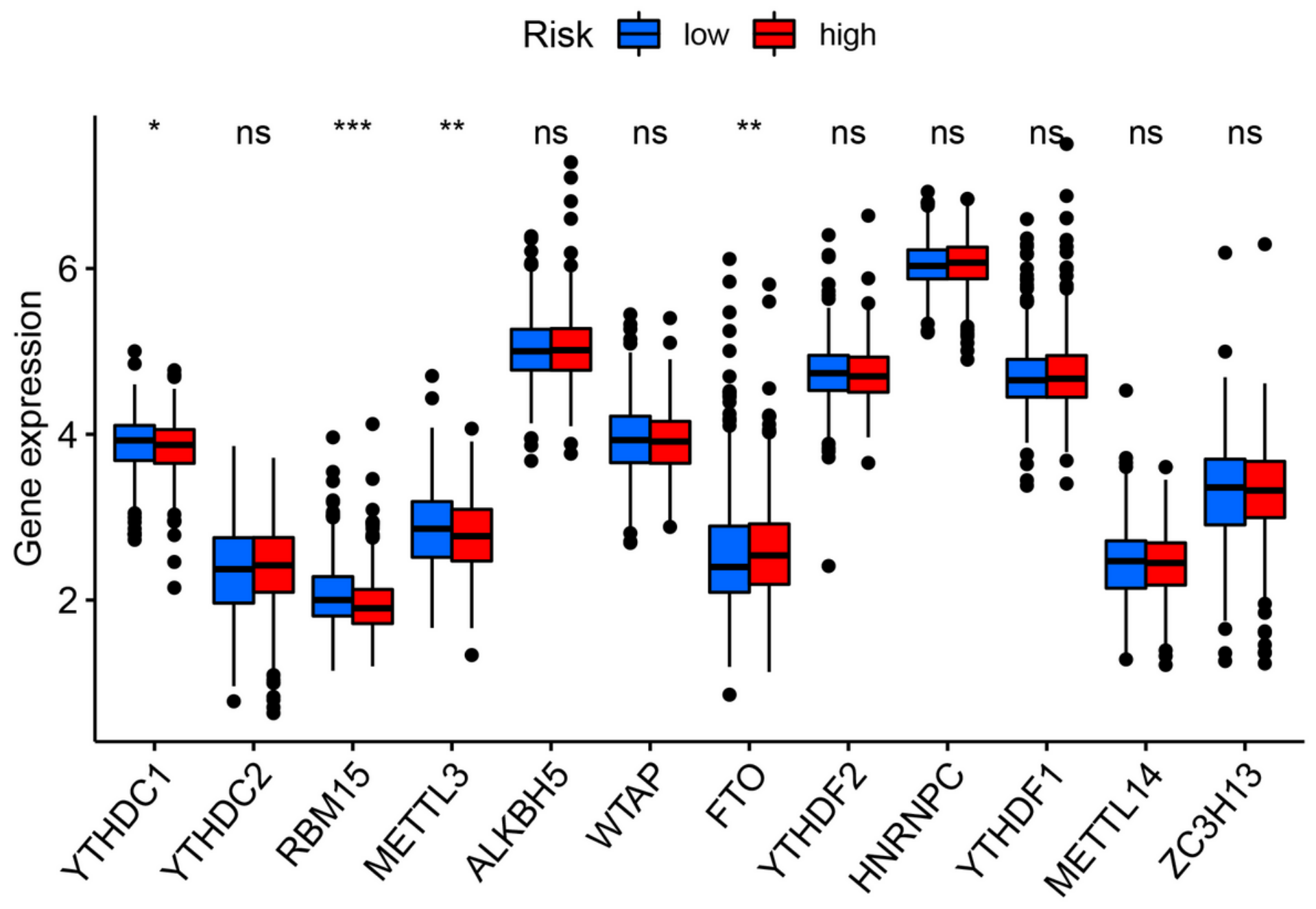

Figure 10

The expression of m6A-related genes between high and low risk group.

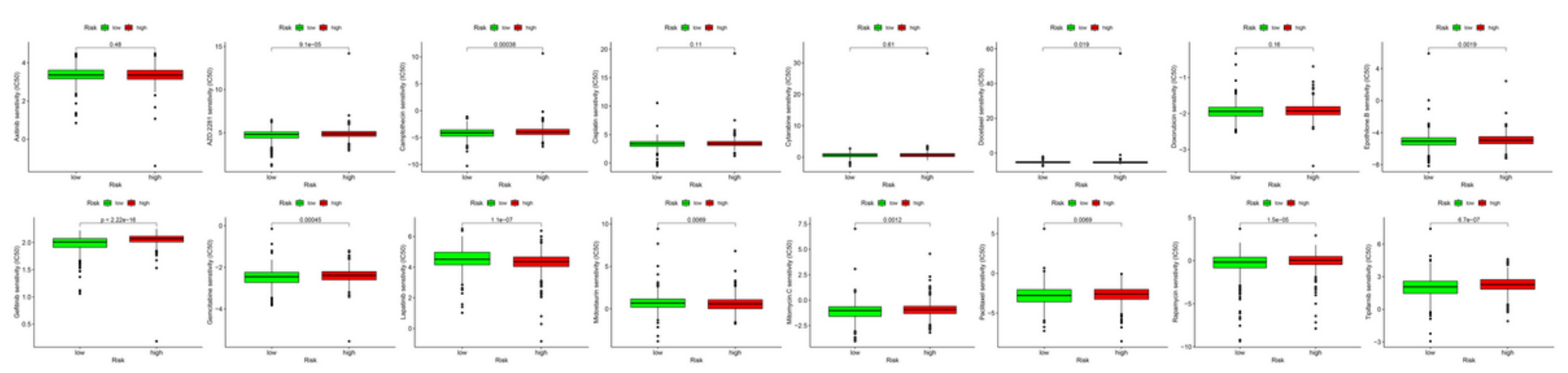

Figure 11

Correlation analysis between the risk model and chemotherapy outcome 


\section{Supplementary Files}

This is a list of supplementary files associated with this preprint. Click to download.

- Supplementarytables.xlsx 